\title{
Searches for light sterile neutrinos with multitrack displaced vertices
}

\author{
Giovanna Cottin \\ Cavendish Laboratory, University of Cambridge, Cambridge CB3 OHE, United Kingdom \\ and Department of Physics, National Taiwan University, Taipei 10617, Taiwan \\ Juan Carlos Helo ${ }^{\dagger}$ \\ Departamento de Física, Facultad de Ciencias, Universidad de La Serena, \\ Avenida Cisternas 1200, La Serena, Chile \\ and Centro-Científico-Tecnológico de Valparaíso, Casilla 110-V, Valparaíso, Chile \\ Martin Hirsch \\ AHEP Group, Instituto de Física Corpuscular - C.S.I.C./Universitat de València, \\ Edificio de Institutos de Paterna, Apartado 22085, E-46071 València, Spain
}

(Received 15 January 2018; published 16 March 2018; corrected 8 March 2019)

\begin{abstract}
We study discovery prospects for long-lived sterile neutrinos at the LHC with multitrack displaced vertices, with masses below the electroweak scale. We reinterpret current displaced vertex searches making use of publicly available, parametrized selection efficiencies for modeling the detector response to displaced vertices. We focus on the production of right-handed $W_{R}$ bosons and neutrinos $N$ in a left-right symmetric model, and find poor sensitivity. After proposing a different trigger strategy (considering the prompt lepton accompanying the neutrino displaced vertex) and optimized cuts in the invariant mass and track multiplicity of the vertex, we find that the LHC with $\sqrt{s}=13 \mathrm{TeV}$ and $300 \mathrm{fb}^{-1}$ is able to probe sterile neutrino masses between $10 \mathrm{GeV}<m_{N}<20 \mathrm{GeV}$ (for a right-handed gauge boson mass of $2 \mathrm{TeV}<m_{W_{R}}<3.5 \mathrm{TeV}$ ). To probe higher masses up to $m_{N} \sim 30 \mathrm{GeV}$ and $m_{W_{R}}<5 \mathrm{TeV}, 3000 \mathrm{fb}^{-1}$ will be needed. This work joins other efforts in motivating dedicated experimental searches to target this low sterile neutrino mass region.
\end{abstract}

DOI: 10.1103/PhysRevD.97.055025

\section{INTRODUCTION}

With the discovery of the Higgs boson [1,2], the Large Hadron Collider (LHC) has confirmed the particle content of the Standard Model (SM). Still one of the main unanswered fundamental questions is the origin of neutrino masses. Experimental data on neutrino oscillations [3] provides clear evidence that there must be new physics beyond the SM, whose effects are actively being looked for by the LHC experiments.

The smallness of neutrino masses may be explained by the so-called seesaw mechanism [4], introducing the existence of massive, right-handed (i.e. sterile) neutrinos [5,6] (see Ref. [7] for a comprehensive review). Different

\footnotetext{
*gcottin@phys.ntu.edu.tw

jchelo@userena.cl

mahirsch@ific.uv.es
}

Published by the American Physical Society under the terms of the Creative Commons Attribution 4.0 International license. Further distribution of this work must maintain attribution to the author(s) and the published article's title, journal citation, and DOI. Funded by SCOAP. realizations of this mechanism give rise to sterile neutrinos with Majorana masses covering various mass ranges (for a more extensive discussion on collider searches and limits, see Refs. [8-10] and references therein). Particularly in leftright symmetric extensions of the SM [11,12], the production and decay of the sterile neutrino $N$ depends mostly on the unknown mass of the new, heavy right-handed gauge boson, $W_{R}$.

In the mass region where $m_{N} \ll m_{W_{R}}$, the distinctive (lepton-number-violating) signature of same-sign dileptons [13] has been extensively studied [8-10,14-22]. If the sterile neutrino mass is also below the electroweak scale (i.e. $m_{N}<m_{W}$ ), it can be long-lived, and travel a measurable distance before decaying inside the LHC detectors.

Different collider searches for long-lived sterile neutrinos have been studied in Refs. [23-35]. At the LHC, these include signatures of trileptons [25,32], lepton jets [29,32], displaced "neutrino jets" [25,30], displaced vertices from Higgs decays [31,33] and displaced vertices, defined in a broad sense [35].

These distinct search proposals target different neutrino mass regions. For example, $13 \mathrm{TeV}$ searches for "neutrino jets" can reach neutrino masses of order hundreds of 
$\mathrm{GeV}$ [30]. In this work we are interested in $\mathcal{O}(10 \mathrm{GeV})$ neutrinos. Displaced lepton-jet searches can place strong limits on sterile neutrinos in the $(4-25 \mathrm{GeV})$ mass range [29]. Here we propose a complementary search for displaced vertices, that has the advantage of zero background.

This work considers a different search strategy, in which events are triggered by a prompt lepton and the neutrino charged decay products are identified to come from a common displaced vertex (DV), where a detailed detector response to displaced vertices is implemented in the form of parametrized selection efficiencies, recently made public by the ATLAS Collaboration [36].

Our search proposal is inspired by the ATLAS multitrack displaced vertex analysis [37,38]. This work particularly expands the previous work in Ref. [35] by considering a smaller fiducial region for displaced decay lengths, in order to have decays limited to the inner trackers of the LHC detectors $(<300 \mathrm{~mm})$ and by accurately modeling the detector response to displaced vertices inside them. In addition, our search strategy differs from the one in Ref. [35] by triggering on the prompt lepton, instead of placing cuts on leptons and/or jets coming from the displaced vertex, and, by reconstructing the displaced vertex position from the neutrino charged decay products (i.e. tracks).

The rest of the paper is structured as follows. We briefly review the phenomenology of left-right symmetric models in Sec. II. In Sec. III we discuss on our proposed DV strategy and discovery prospects at the LHC. Concluding remarks are presented in Sec. IV.

\section{LEFT-RIGHT SYMMETRIC MODEL}

The model considered is the left-right symmetric extension of the SM [11,12,39], with gauge group $S U(2)_{L} \times$ $S U(2)_{R} \times U(1)_{B-L}$ and couplings $g_{L}, g_{R}, g_{1}$, respectively. This model contains a right-handed gauge boson $W_{R}$ and three right-handed Majorana neutrinos, with lightest state $N$. In the mass range we are interested in (i.e. $m_{N} \ll m_{W_{R}}$, $m_{N}<m_{W}$ ), the sterile neutrino proper decay length can be written as $[35,40,41]$

$$
c \tau_{N} \sim 0.12\left(\frac{10 \mathrm{GeV}}{m_{N}}\right)^{5}\left(\frac{m_{W_{R}}}{1000 \mathrm{GeV}}\right)^{4}[\mathrm{~mm}] .
$$

A diagram showing sterile neutrino production and decay at the LHC can be seen in Fig. 1.

This process leads to different signatures, depending on the neutrino lifetime and mass. Existing searches by ATLAS [42] and CMS [43] place a lower limit on $m_{W_{R}}$ at $3 \mathrm{TeV}$, for $0.2 \mathrm{TeV} \lesssim m_{N} \lesssim 2 \mathrm{TeV}$. CMS has recently excluded right-handed bosons with masses $m_{W_{R}}<4.4 \mathrm{TeV}$, for $m_{N}=m_{W_{R}} / 2$ [44]. Searches for new resonances in the dijet distribution can also be used to place limits on right-handed bosons [45-47], roughly of order $3 \mathrm{TeV}$. For neutrino decays

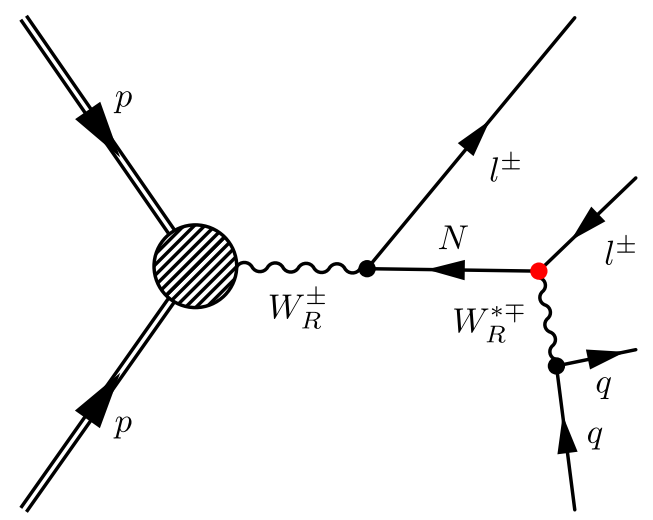

FIG. 1. Sterile neutrino $N$ production and decay at the LHC in the left-right symmetric model. For $m_{N} \ll m_{W_{R}}$, production proceeds through a $W_{R}$, followed by $N$ displaced decay to a lepton and quarks. The displaced vertex position is represented by the red circle.

outside of the detector, searches for new heavy gauge bosons decaying to leptons and missing transverse momentum $[48,49]$ can be sensitive.

Early constraints on left-right symmetric models from LHC data have been addressed in Ref. [50]. The very recent study in Ref. [51] tried to systematically asses constraints covering the entire neutrino mass range, and explored sensitivity from different searches. For decays in the "displaced region" in the authors' definition, displaced jets searches (see for example Ref. [52]) can be effective.

Currently, no public searches at the LHC target sterile neutrinos with masses as low as a few GeV.

In the rest of the paper we restrict our discussion to sterile neutrino mixing with the electron sector only, for simplicity.

\section{SIMULATION AND RESULTS}

We generate a UFO [53] model with SARAH [54] and use SPHENO $[55,56]$ for the spectrum calculation of the left-right symmetric model. The SARAH model files are taken from the implementation of the left-right symmetric model given in [57]. We simulate events for the process $p p \rightarrow W_{R}^{ \pm} \rightarrow N e^{ \pm}$. Generation is performed with MADGRAPH5_AMC@NLOv2.4.3 [58] at leading order. The output corresponds to unweighted events in LHE format [59]. The $N$ lifetimes are included using the time_of_flight option in MADGRAPH5.

The generated events are then interfaced to PYTHIA8 v2.15 [60] for hadronization and computation of the $N$ decays. We use FASTJET 3.1.3 [61] for jet and trackless jet reconstruction. Lepton reconstruction and the identification of the displaced vertices is also done inside PYTHIA8. The masses and decay widths of the particles in the model are communicated to PYTHIA via the SLHA $[62,63]$ section of the LHE header. Plots are generated with matplotlib [64]. 
The ATLAS analysis in Ref. [38] looks for high track-multiplicity displaced vertices at $13 \mathrm{TeV}$ in events possessing displaced vertices and missing transverse momenta. We follow the detailed prescription using parametrized selection efficiencies as a function of displaced vertex radial distance, number of tracks and mass, that can be applied to vertices passing certain particle-level acceptance requirements using the truth Monte Carlo event record. These efficiencies can be found in Ref. [36], and are given for different regions in the detector, encapsulating also the effect of the material veto cut the analysis implements to remove background vertices. ${ }^{1}$

The analysis in Ref. [38] originally triggered on missing transverse momenta in the event that was bigger than $250 \mathrm{GeV}$. In this work, we propose to trigger on the prompt lepton coming from the $W_{R}$ decay, ${ }^{2}$ as in our model there is little or no missing transverse momenta. Apart from the trigger requirement, we fully recast the multitrack analysis in Ref. [38].

We require events to satisfy the following selections.

(1) Prompt electron: One electron ${ }^{3}$ with $p_{T}>25 \mathrm{GeV}$.

(2) Trackless jet: One "trackless jet" with $p_{T}>70 \mathrm{GeV}$, or two trackless jets with $p_{T}>25 \mathrm{GeV}$. A trackless jet is defined as a jet for which the scalar sum of the $p_{T}$ of all charged particles inside the jet does not exceed $5 \mathrm{GeV}^{4}$

In addition, each event must have at least one displaced vertex with the following.

(3) DV fiducial: Distance between the interaction point and the decay position $>4 \mathrm{~mm}$. The decay position must also lie in the fiducial region $r_{\mathrm{DV}}<300 \mathrm{~mm}$ and $\left|z_{\mathrm{DV}}\right|<300 \mathrm{~mm}$.

(4) DV $N_{\text {trk }}$ : The number of selected decay products must be at least five, where selected decay products

\footnotetext{
${ }^{1}$ The parametrized efficiencies provided by the ATLAS analysis were validated in Refs. [65,66].

${ }^{2}$ The ATLAS $8 \mathrm{TeV}$ [37] version of this analysis considers a DV + lepton signature, where the lepton that fires the trigger is associated with the displaced vertex. Here we do not follow this approach, as the decay products of the displaced $N$ are too collimated for the displaced lepton to satisfy isolation requirements. Alternatively, a "trackless jet trigger" [67]— where a jet matched with a muon is required-could be used to trigger on events, although it was shown that this trigger is inefficient for reconstructing displaced vertices within the tracker [68].

${ }^{3}$ Note that in our simplified model we consider mixing in the electron sector only, so the prompt lepton is an electron. In the case where muon mixing is present, a muon trigger can be used.

${ }^{4}$ This requirement is applied offline when processing data on disk, and is part of the first filtering in Ref. [38] that gives the data set where the large-radius tracking is applied to. Therefore, this trackless jet cut can only see prompt tracks, so a particle decaying inside the tracker can pass this selection.
}

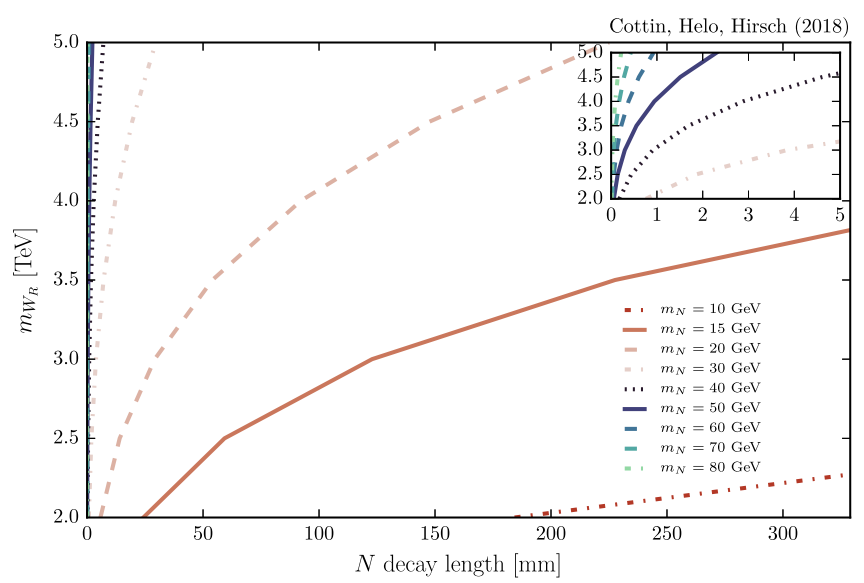

FIG. 2. Sterile neutrino decay distance as a function of $W_{R}$ mass, for fixed values of the sterile neutrino mass. The upper right plot shows a zoomed region, where higher neutrino mass curves can be seen.

are charged and stable, with $p_{T}>1 \mathrm{GeV}$ and transverse impact parameter $\left|d_{0}\right|>2 \mathrm{~mm}$.

(5) DV $m_{\mathrm{DV}}$ : The invariant mass of the truth vertex must be larger than $10 \mathrm{GeV}$, and is constructed assuming all charged decay products have the mass of the pion.

(6) DV efficiency: Parametrized selection efficiencies are implemented depending on the displaced vertex distance, number of tracks and mass, following the prescription in Ref. [36].

Based on these cuts, we first study the neutrino masses the analysis can be sensitive to. We simulate a grid of points with $m_{N}=[10-80] \mathrm{GeV}$ and $m_{W_{R}}=[2-5] \mathrm{TeV}$. At each point we calculate the neutrino decay length, given by $c \tau_{N}\langle\beta \gamma\rangle$, where the average of the $\beta \gamma$ factor is taken after 10000 events, and it is roughly $\mathcal{O}(100)$ for the scanned grid.

Figure 2 shows the dependence on the decay position with the $W_{R}$ mass, for fixed values of the sterile neutrino mass. We note that for $m_{N}>40 \mathrm{GeV}$, decays are below $4 \mathrm{~mm}$, and will therefore fail to be in the fiducial region required by the analysis. An optimal acceptance region lies between $10 \mathrm{GeV}<m_{N}<40 \mathrm{GeV}$ and $2 \mathrm{TeV}<m_{W_{R}}<$ $5 \mathrm{TeV}$.

We therefore choose a particular benchmark point with $m_{N}=20 \mathrm{GeV}$ and $m_{W_{R}}=4 \mathrm{TeV}$, and proper neutrino decay distance $c \tau_{N}=1.3 \mathrm{~mm}$. We show the effect of applying all analysis cuts on this benchmark in Table I.

We see very low efficiency, reaching $0.06 \%$ in Table I. The loss comes mainly from the last two cuts $-N_{\text {trk }} \geq 5$ and invariant vertex mass $m_{\mathrm{DV}}>10 \mathrm{GeV}$-which are too restrictive for the given neutrino mass of $m_{N}=20 \mathrm{GeV}$, as softer decay products will lead to a smaller amount of tracks available to make up a vertex. This sensitivity loss was also noted in a model with a long-lived singlino 
TABLE I. Numbers of simulated events $N$ at $\sqrt{s}=13 \mathrm{TeV}$, relative and overall efficiencies for our left-right model with $m_{N}=20 \mathrm{GeV}, m_{W_{R}}=4 \mathrm{TeV}$ and $c \tau_{N}=1.3 \mathrm{~mm}$, for the ATLAS default cuts.

\begin{tabular}{lrcc}
\hline \hline & $N$ & Rel. $\epsilon[\%]$ & Ov. $\epsilon[\%]$ \\
\hline All events & 10000 & 100 & 100 \\
Prompt electron & 8721 & 87.2 & 87.2 \\
Trackless jet & 8704 & 99.8 & 87.0 \\
DV fiducial & 7615 & 87.5 & 76.1 \\
DV $N_{\text {trk }}$ & 528 & 6.9 & 5.3 \\
DV $m_{\text {DV }}$ & 89 & 16.9 & 0.9 \\
DV efficiency & 6 & 6.7 & 0.06 \\
\hline \hline
\end{tabular}

decaying to a light ( 20 GeV) pseudoscalar [69], and in a model with displaced Higgs decays to light $(\sim 10 \mathrm{GeV})$ hidden scalars [68].

We attempt to improve the sensitivity by loosening the $N_{\text {trk }}$ and $m_{\mathrm{DV}}$ cuts, requiring $N_{\text {trk }}>3$ and $m_{\mathrm{DV}} \geq 5 \mathrm{GeV}$.

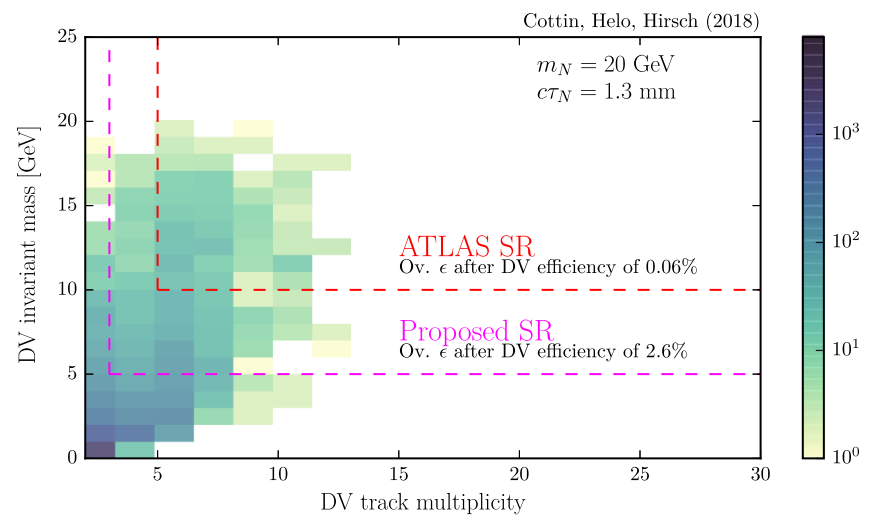

FIG. 3. Invariant mass of the displaced vertex against number of tracks for our left-right model with $m_{N}=20 \mathrm{GeV}, m_{W_{R}}=$ $4 \mathrm{TeV}$ and $c \tau_{N}=1.3 \mathrm{~mm}$. Our proposed signal region is defined by the $N_{\text {trk }}>3$ and $m_{\mathrm{DV}} \geq 5 \mathrm{GeV}$ cuts.

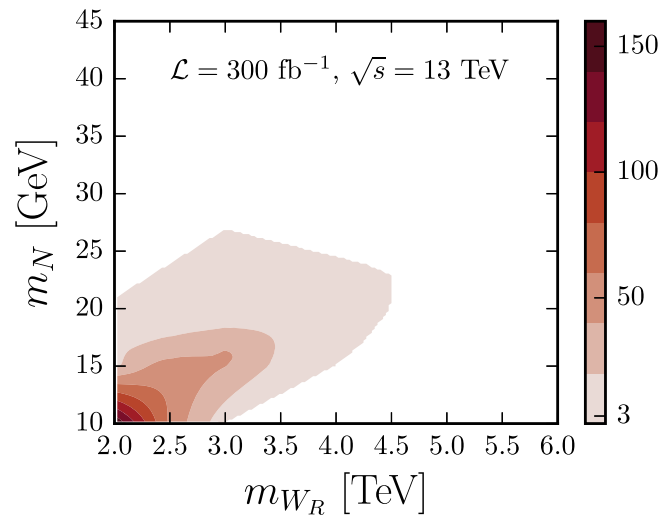

We should note that, even though the backgrounds to the multitrack displaced vertex search come mostly from instrumental sources (including hadronic interactions in dense material regions and random crossing of tracks), lowering the invariant mass and track multiplicity cuts could in principle increase background rates. Nevertheless, we expect these to still be within negligible levels with the requirements of displaced activity (i.e. $d_{0}$ and trackless jet cut). In addition, there are still no background yields reported by ATLAS in this relaxed region, as it can be seen in Fig. 7 of Ref. [38]. Of course, a dedicated experimental background estimation study with these optimized cuts would be needed, which is beyond the capabilities of our detector simulations.

Figure 3 shows the invariant mass of the displaced vertex against the number of tracks for our benchmark. Prompt electron, Trackless jet and DV fiducial cuts are applied (cuts 1. to 3.). The last two cuts of DV $N_{\text {trk }}$ and DV $m_{\mathrm{DV}}$ (cuts 4 . and 5.) define the regions where signal is expected to be found (SR) and are shown as dashed boxes in the figure. The red box defines a SR with the default ATLAS cuts of $N_{\text {trk }} \geq 5$ and $m_{\mathrm{DV}}>10 \mathrm{GeV}$. The purple box defines our proposed SR with $N_{\text {trk }}>3$ and $m_{\mathrm{DV}} \geq 5 \mathrm{GeV}$. In each boxed region we show the final efficiency obtained after applying the experimental DV efficiency (cut 6.). We see an efficiency improvement of 2 orders of magnitude in our proposed SR.

With the proposed tuned cuts of $N_{\text {trk }}>3$ and $m_{\mathrm{DV}} \geq$ $5 \mathrm{GeV}$ we now discuss discovery prospects. Figure 4 shows the number of signal events in the mass plane $m_{W_{R}}-m_{N}$. Regions producing at least three signal events are shown, which is reasonable to set as a requirement for discovery in the absence of background. We see in the left plot of Fig. 4 that at $13 \mathrm{TeV}$ and with $300 \mathrm{fb}^{-1}$ the LHC is sensitive to masses up to $m_{N} \sim 20 \mathrm{GeV}$, for $m_{W_{R}} \sim 3.5 \mathrm{TeV}$. With a higher luminosity of $3000 \mathrm{fb}^{-1}$ in the right plot, the $13 \mathrm{TeV}$ LHC can reach sterile neutrino masses up to $\sim 30 \mathrm{GeV}$ for similar $W_{R}$ masses.

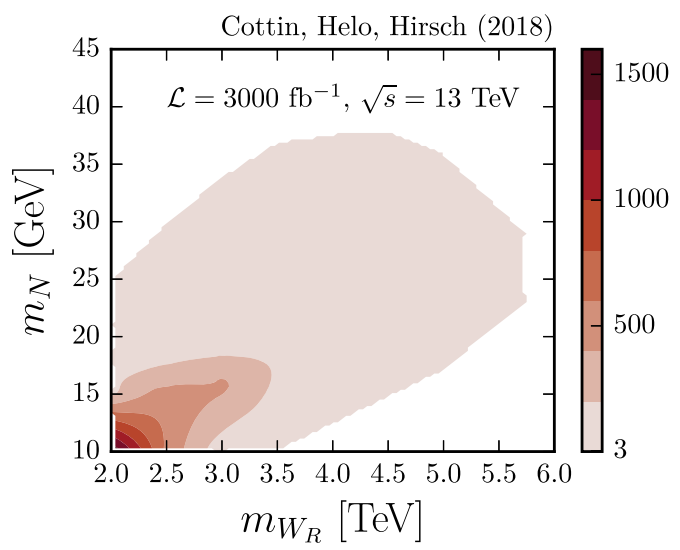

FIG. 4. Number of signal events for our left-right model at $\sqrt{s}=13 \mathrm{TeV}$, with our proposed search strategy, with $\mathcal{L}=300 \mathrm{fb}^{-1}($ left) and $\mathcal{L}=3000 \mathrm{fb}^{-1}$ (right). 


\section{SUMMARY AND CONCLUSIONS}

We have studied the sensitivity of current multitrack displaced vertex searches at the LHC for probing longlived, light sterile neutrinos. We based our study on a leftright symmetric model, and considered sterile neutrino masses $m_{N} \ll m_{W_{R}}$, and $m_{N}<m_{W}$.

We found that for $40 \mathrm{GeV}<m_{N}<80 \mathrm{GeV}$, neutrino decay distances are below $4 \mathrm{~mm}$, and will therefore fail to be in the analysis fiducial tracker region.

After considering a different trigger strategy on the prompt lepton in the event, we found very poor signal efficiency for events passing the standard DV cuts. This is due to the low mass of the sterile neutrino, as it is too soft to produce much tracks.

After loosening the DV invariant mass and number of tracks to a region where still zero background is reported [38], we found that with $3000 \mathrm{fb}^{-1}$ of integrated luminosity at $13 \mathrm{TeV}$, this proposed "loose multitrack $\mathrm{DV}+$ prompt lepton" search should be sensitive to sterile neutrino masses up to $\sim 30 \mathrm{GeV}$.

The identification of displaced vertices will continue to be an important signature of new physics, given that this signal is scarce in the SM. We encourage the experimental collaborations at the LHC to pursue these searches further, particularly in the study of lower cuts in the displaced vertex invariant mass, as $m_{N}$ lower than $10 \mathrm{GeV}$ may be probed.

\section{ACKNOWLEDGMENTS}

G. C. thanks Christian Ohm for helpful discussions on the ATLAS multitrack displaced vertex analysis. The work of G.C. while at Cambridge was funded by the postgraduate Conicyt-Chile/Cambridge Trusts Scholarship 84130011, where this work was initiated. G. C. is now supported by the Ministry of Science and Technology of Taiwan under Grant No. MOST-106-2811M-002-035. J. C. H. is supported by Chile grants Fondecyt No. 1161463, Conicyt PIA/ACT 1406 and Basal FB0821. M.H. was funded by Spanish MICINN Grants No. FPA2017-85216-P and No. SEV-2014-0398 (from the Ministerio de Economía, Industria y Competitividad), as well as PROMETEOII/2014/084 (from the Generalitat Valenciana).
[1] G. Aad et al. (ATLAS Collaboration), Phys. Lett. B 716, 1 (2012).

[2] S. Chatrchyan et al. (CMS Collaboration), Phys. Lett. B 716, 30 (2012).

[3] K. A. Olive et al. (Particle Data Group), Chin. Phys. C 38, 090001 (2014).

[4] P. Minkowski, Phys. Lett. B 67, 421 (1977).

[5] R. N. Mohapatra and G. Senjanovic, Phys. Rev. Lett. 44, 912 (1980).

[6] J. Schechter and J. W. F. Valle, Phys. Rev. D 22, 2227 (1980).

[7] K. N. Abazajian et al., arXiv:1204.5379.

[8] Y. Cai, T. Han, T. Li, and R. Ruiz, arXiv:1711.02180.

[9] F. F. Deppisch, P. S. Bhupal Dev, and A. Pilaftsis, New J. Phys. 17, 075019 (2015).

[10] A. Atre, T. Han, S. Pascoli, and B. Zhang, J. High Energy Phys. 05 (2009) 030.

[11] J. C. Pati and A. Salam, Phys. Rev. D 10, 275 (1974); 11, 703(E) (1975).

[12] R. N. Mohapatra and J. C. Pati, Phys. Rev. D 11, 2558 (1975).

[13] W.-Y. Keung and G. Senjanovic, Phys. Rev. Lett. 50, 1427 (1983).

[14] A. Datta, M. Guchait, and A. Pilaftsis, Phys. Rev. D 50, 3195 (1994).

[15] O. Panella, M. Cannoni, C. Carimalo, and Y. N. Srivastava, Phys. Rev. D 65, 035005 (2002).

[16] T. Han and B. Zhang, Phys. Rev. Lett. 97, 171804 (2006).
[17] F. del Aguila, J. A. Aguilar-Saavedra, and R. Pittau, J. High Energy Phys. 10 (2007) 047.

[18] L. Basso, A. Belyaev, S. Moretti, and C. H. ShepherdThemistocleous, Phys. Rev. D 80, 055030 (2009).

[19] P. Fileviez Perez, T. Han, and T. Li, Phys. Rev. D 80, 073015 (2009).

[20] J. N. Ng, A. de la Puente, and B. W.-P. Pan, J. High Energy Phys. 12 (2015) 172.

[21] P. Cox, C. Han, and T. T. Yanagida, J. High Energy Phys. 01 (2018) 037.

[22] A. Das, P. Konar, and A. Thalapillil, J. High Energy Phys. 02 (2018) 083.

[23] C. O. Dib, C. S. Kim, N. A. Neill, and X.-B. Yuan, Phys. Rev. D 97, 035022 (2018).

[24] S. Antusch, E. Cazzato, and O. Fischer, Phys. Lett. B 774, 114 (2017).

[25] E. Accomando, L. Delle Rose, S. Moretti, E. Olaiya, and C. H. Shepherd-Themistocleous, J. High Energy Phys. 02 (2018) 109.

[26] P. S. B. Dev, R. N. Mohapatra, and Y. Zhang, Nucl. Phys. B923, 179 (2017).

[27] S. Antusch, E. Cazzato, and O. Fischer, J. High Energy Phys. 12 (2016) 007.

[28] S. Antusch, E. Cazzato, and O. Fischer, Int. J. Mod. Phys. A 32, 1750078 (2017).

[29] S. Dube, D. Gadkari, and A. M. Thalapillil, Phys. Rev. D 96, 055031 (2017).

[30] M. Mitra, R. Ruiz, D. J. Scott, and M. Spannowsky, Phys. Rev. D 94, 095016 (2016). 
[31] E. Accomando, L. Delle Rose, S. Moretti, E. Olaiya, and C. H. Shepherd-Themistocleous, J. High Energy Phys. 04 (2017) 081.

[32] E. Izaguirre and B. Shuve, Phys. Rev. D 91, 093010 (2015).

[33] A. M. Gago, P. Hernández, J. Jones-Pérez, M. Losada, and A. Moreno Briceño, Eur. Phys. J. C 75, 470 (2015).

[34] C. Dib and C. S. Kim, Phys. Rev. D 89, 077301 (2014).

[35] J. C. Helo, M. Hirsch, and S. Kovalenko, Phys. Rev. D 89, 073005 (2014); 93, 099902(E) (2016).

[36] https://atlas.web.cern.ch/Atlas/GROUPS/PHYSICS/PAPERS/ SUSY-2016-08/hepdata_info.pdf.

[37] G. Aad et al. (ATLAS Collaboration), Phys. Rev. D 92, 072004 (2015).

[38] M. Aaboud et al. (ATLAS Collaboration), arXiv:1710. 04901.

[39] R. N. Mohapatra and G. Senjanovic, Phys. Rev. D 23, 165 (1981).

[40] O. Castillo-Felisola, C. O. Dib, J. C. Helo, S. G. Kovalenko, and S. E. Ortiz, Phys. Rev. D 92, 013001 (2015).

[41] A. Maiezza, M. Nemevšek, and F. Nesti, Phys. Rev. Lett. 115, 081802 (2015).

[42] G. Aad et al. (ATLAS Collaboration), J. High Energy Phys. 07 (2015) 162.

[43] V. Khachatryan et al. (CMS Collaboration), Eur. Phys. J. C 74, 3149 (2014).

[44] CMS Collaboration, Search for a heavy right-handed $W$ boson and a heavy neutrino in events with two sameflavor leptons and two jets at $\sqrt{(s)}=13 \mathrm{TeV}$, Tech. Report No. CMS-PAS-EXO-17-011, 2017).

[45] G. Aad et al. (ATLAS Collaboration), Phys. Lett. B 754, 302 (2016).

[46] V. Khachatryan et al. (CMS Collaboration), Phys. Rev. Lett. 116, 071801 (2016).

[47] M. Aaboud et al. (ATLAS Collaboration), Phys. Rev. D 96, 052004 (2017).

[48] S. Chatrchyan et al. (CMS Collaboration), Phys. Lett. B 701, 160 (2011).

[49] M. Aaboud et al. (ATLAS Collaboration), arXiv:1706 .04786 .
[50] M. Nemevsek, F. Nesti, G. Senjanovic, and Y. Zhang, Phys. Rev. D 83, 115014 (2011).

[51] M. Nemevšek, F. Nesti, and G. Popara, arXiv:1801.05813.

[52] V. Khachatryan et al. (CMS Collaboration), Phys. Rev. D 91, 012007 (2015).

[53] C. Degrande, C. Duhr, B. Fuks, D. Grellscheid, O. Mattelaer, and T. Reiter, Comput. Phys. Commun. 183, 1201 (2012).

[54] F. Staub, Comput. Phys. Commun. 185, 1773 (2014).

[55] W. Porod and F. Staub, Comput. Phys. Commun. 183, 2458 (2012).

[56] W. Porod, Comput. Phys. Commun. 153, 275 (2003).

[57] C. Bonilla, M.E. Krauss, T. Opferkuch, and W. Porod, J. High Energy Phys. 03 (2017) 027.

[58] J. Alwall, R. Frederix, S. Frixione, V. Hirschi, F. Maltoni, O. Mattelaer, H. S. Shao, T. Stelzer, P. Torrielli, and M. Zaro, J. High Energy Phys. 07 (2014) 079.

[59] J. Alwall et al., Comput. Phys. Commun. 176, 300 (2007).

[60] T. Sjöstrand, S. Ask, J. R. Christiansen, R. Corke, N. Desai, P. Ilten, S. Mrenna, S. Prestel, C. O. Rasmussen, and P.Z. Skands, Comput. Phys. Commun. 191, 159 (2015).

[61] M. Cacciari, G. P. Salam, and G. Soyez, Eur. Phys. J. C 72, 1896 (2012).

[62] P. Z. Skands et al., J. High Energy Phys. 07 (2004) 036.

[63] B. C. Allanach et al., Comput. Phys. Commun. 180, 8 (2009).

[64] J. D. Hunter, Computing In Science \& Engineering 9, 90 (2007).

[65] LHC-LLP community white paper (to be published).

[66] Les Houches Working Group report (to be published).

[67] G. Aad et al. (ATLAS Collaboration), J. Inst. 8, P07015 (2013).

[68] C. Csaki, E. Kuflik, S. Lombardo, and O. Slone, Phys. Rev. D 92, 073008 (2015).

[69] B. C. Allanach, M. Badziak, G. Cottin, N. Desai, C. Hugonie, and R. Ziegler, Eur. Phys. J. C 76, 482 (2016).

Correction: The previously published Figure 4 contained an error and has been replaced. 